\title{
CLASSIFYING SPACES AND INFINITE SYMMETRIC PRODUCTS
}

\author{
BY \\ M. C. MCCORD( $\left.{ }^{1}\right)$
}

1. Introduction. The object of study in this paper is a construction $B(G, X)$ which essentially includes the classifying space construction $B_{G}$ of Milgram [7] and Steenrod [12] and the infinite symmetric product construction $\operatorname{SP}(X)$ of Dold and Thom [4] as special cases.

$\$ \S 2,3$, and 4 are preliminary in nature. Let us just remark that $\$ 2$ describes the category of spaces we are working in (a modification of Steenrod's [11]). We make the convention that in the rest of the paper all spaces, products, topological monoids, etc., are meant in the sense of this category.

$\$ 5$ deals with the algebraic and set-theoretic side of the construction $B(\cdot, \cdot)$. The definition is quite simple: If $G$ is a monoid with unit $e$ and $X$ is a based set, then $B(G, X)$ is the monoid of all functions $u: X \rightarrow G$ such that $u(*)=e$ and such that $u(x)=e$ for all but finitely many $x \in X$.

In $\S 6$ we topologize $B(G, X)$ when $G$ is an abelian topological monoid and $X$ is a based space, in such a way that $B(\cdot, \cdot)$ becomes a bifunctor to the category of abelian topological monoids. If $G$ is an abelian topological group, then so is $B(G, X)$.

This construction includes the following special cases (up to topological isomorphism): (1) $\left(B\left(G, S^{0}\right) \approx G\right.$. (2) $B\left(G, S^{1}\right) \approx B_{G}$ as in [7] or [12]. (3) $B(G, I) \approx E_{G}$ as in [7] or [12]. (4) If $G$ is a discrete abelian group, then $B\left(G, S^{n}\right)$ is an EilenbergMacLane space $K(G, n)$ (see $\S 10$ ). (5) If $Z^{+}$is the additive monoid of nonnegative integers, then $B\left(Z^{+}, X\right) \approx S P(X)$ as in [4]. (6) If $\mathbf{Z}$ is the additive group of integers, then $B(Z, X) \approx A G(X)$, defined in [4] as a certain quotient space of $\operatorname{SP}(X \vee X)$. (7) $B(\mathbf{Z} / m \mathbf{Z}, X) \approx A G(X ; m)$, defined in [4] as $A G(X) / m A G(X)$. (8) One could form topological monoids such as $B(S P(X), Y)$, or more generally $B(B(G, X), Y)$. In 6.13 it is seen that the latter is topologically isomorphic to $B(G, X \wedge Y)$.

In $\$ 7$ we generalize a result of Dold and Thom [4] by showing that $B(G, X)$ is a CW complex whenever $G$ is a discrete abelian monoid and $X$ is triangulable.

For a fixed abelian topological monoid [group] $G$, the functor $B(G, \cdot)$ has a tendency to convert cofibrations $A \rightarrow X \rightarrow X / A$ to quasifibrations [fibrations]

$$
B(G, A) \rightarrow B(G, X) \rightarrow B(G, X / A) .
$$

Received by the editors May 28, 1968.

(1) This research was partially supported by National Science Foundation Grant GP$7952 X$. 
In $\S 8$ we give a theorem in this direction (see Theorem 8.8 together with Remark 8.9), generalizing one of the theorems of Dold and Thom [4]. The natural filtration of $B(G, \cdot)$ (see $(5.1)$ and $(6.1)$ ), which figures in the proof, generalizes filtrations used in [7] and [12], but does not correspond to filtrations used in [4]. Part of the proof is abstracted into $\$ 4$.

If $G$ is an arbitrary (not necessarily abelian) topological monoid, the sequence (1.1) still exists set-theoretically, as will be seen from $\$ 5$. When can one topologize it appropriately? In $\$ 9$ we do this for the simplest nontrivial pair, $(X, A)=\left(I, S^{0}\right)$. This topologization is consistent with that of $\S 6$ if $G$ is abelian (see 9.18). Since $B\left(G, S^{0}\right) \approx G$ (algebraically), we actually view the sequence as

$$
G \rightarrow B(G, I) \rightarrow B\left(G, S^{1}\right) .
$$

This sequence is homeomorphic to the sequence $G \rightarrow E_{G} \rightarrow B_{G}$ constructed by Milgram [7] or Steenrod [12]. We do not actually prove the equivalence, but we set up enough basic properties of (1.2) that one could easily do so. Setting up these properties costs little extra effort, because the method for topologizing $B(G, I)$ and $B\left(G, S^{1}\right)$ is very close to the general method of $\S 6$ for topologizing $B(G, X)$ when $G$ is abelian-with the result that $\$ 9$ is almost a special case of $\$ \S 6$ and 8 . In particular, the proof of the fibration theorem of $\S 8$ requires only minor modifications to prove the following (9.17): If $G$ is a topological group such that $\{e\} \rightarrow G$ is a cofibration, then (1.2) is a numerable principal $G$-bundle. Numerability (in the sense of Dold [3]) is not shown in [7] or [12].

In $\S 10$ we discuss canonical maps $B(G, X) \rightarrow \Omega B(G, S X)$, both in the context of $\S 6$ with $G$ abelian, and in the context of $\S 9$ with $X=S^{0}$ (the latter being related to $[7, \S 5])$. Under suitable restrictions these maps are shown to be isomorphisms of $H$-spaces. (We get them to be actual homotopy equivalences, not just weak homotopy equivalences.) An immediate consequence is that if $G$ is a discrete abelian group, then $B\left(G, S^{n}\right)$ is an Eilenberg-MacLane space $K(G, n)$.

In the last section $(\S 11)$ we study the groups of based homotopy classes $[X, B(G, Y)]$, where $X$ and $Y$ are based spaces and $G$ is a discrete abelian group. If $G$ is also a module, then $B(G, Y)$ is a topological module, so that $[X, B(G, Y)]$ is a module. Note that by the preceding paragraph,

$$
\left[X, B\left(G, S^{n}\right)\right] \approx \tilde{H}^{n}(X ; G)
$$

for nice enough $X$. Dually we get (see 11.4)

$$
\left[S^{n}, B(G, Y)\right] \approx \tilde{H}_{n}(Y ; G)
$$

for nice enough $Y$, generalizing one of the main theorems of Dold and Thom [4].

Perhaps one could use the modules $[X, B(G, Y)]$ fruitfully as a definition of homology and cohomology in a "from scratch" development of algebraic topology. This point of view is emphasized in $\$ 11$. In an entirely direct and elementary way, 
we define generalized cross products in the modules $[X, B(G, Y)]$. First we define continuous bilinear maps

$$
B(G, Y) \times B\left(G^{\prime}, Y^{\prime}\right) \rightarrow B\left(G \otimes G^{\prime}, Y \wedge Y^{\prime}\right),
$$

and then these give directly the required products

$$
[X, B(G, Y)] \times\left[X^{\prime}, B\left(G^{\prime}, Y^{\prime}\right)\right] \rightarrow\left[X \wedge X^{\prime}, B\left(G \otimes G^{\prime}, Y \wedge Y^{\prime}\right)\right] .
$$

We end the section by proving in a simple and rather geometric fashion a commutativity law for (1.6) which includes skew commutativity for the case of homology (1.4) and of cohomology (1.3). The pairing (1.5) figures in a homotopical development of the Steenrod operations, which however is not treated in this paper.

I am grateful to Andreas Dress and N. E. Steenrod for useful discussions, and to J. C. Moore for most of the information in $\$ 2$.

2. Compactly generated spaces. The paper [11] of Steenrod shows why it is convenient to work in the category of compactly generated Hausdorff spaces. In some contexts, though, the requirement of the Hausdorff condition can be a problem, because certain standard operations on spaces can lead outside the category. For example, if $(X, A)$ is a closed pair of spaces in the category, $X / A$ may not be in the category. There is a more general problem for adjunction spaces, and a problem for unions of expanding sequences. In the presence of certain extra conditions (see [11]) one can be guaranteed that the spaces remain in the category. But in some situations these conditions may be absent or difficult to verify. I have learned from J. C. Moore how one can slightly enlarge the category so that it has better closure properties, but still has the convenient technical properties in [11]. We proceed as follows.

When we call a space compact we follow the French usage and include the Hausdorff property. A subset $A$ of a space $X$, is compactly closed, if for every map $\varphi: K \rightarrow X$, where $K$ is a compact space, $\varphi^{-1} A$ is closed in $K$. A space $X$ is called a $k$-space if every compactly closed subset of $X$ is closed.

A space $X$ is a weak Hausdorff space if for every map $\varphi: K \rightarrow X$, where $K$ is a compact space, $\varphi K$ is closed in $X$. This property of spaces is between $T_{1}$ and $T_{2}$. It is stable under the formation of cartesian products and subspaces.

2.1. LEMMA. If $X$ is a weak Hausdorff space, then for every map $\phi$ of a compact space $K$ into $X$, the image $\varphi K$ is compact.

Proof. The main point of course is that $\varphi K$ is Hausdorff. If $x_{1}$ and $x_{2}$ are distinct points of $\varphi K$, then from the facts that $X$ is $T_{1}$ and $K$ is normal, we can get disjoint open sets $U_{1}$ and $U_{2}$ containing $\varphi^{-1} x_{1}$ and $\varphi^{-1} x_{2}$, respectively. Since $X$ is weak Hausdorff, the sets $\varphi K-\varphi\left(K-U_{1}\right)$ and $\varphi K-\varphi\left(K-U_{2}\right)$ are open in $\varphi K$; they are disjoint and contain $x_{1}$ and $x_{2}$, respectively.

Note that as a result of this lemma, a subset of a weak Hausdorff space $X$ is 
compactly closed if and only if its intersection with every compact subset of $X$ is closed.

Let us call a space compactly generated if it is a weak Hausdorff $k$-space. The category of compactly generated spaces is the full subcategory of the category of topological spaces whose objects are the compactly generated spaces. This is the category in which we wish to work. It is slightly larger than the category of compactly generated Hausdorff spaces studied in [11]. Nevertheless one can carry over essentially all the ideas of [11].

In particular, if $X$ is a topological space, let $k X$ denote the space whose underlying set is that of $X$ and whose closed sets are the compactly closed subsets of $X$. If $f: X \rightarrow Y$ is a map, we let $k f: k X \rightarrow k Y$ be the map whose underlying function is that of $f$. Then $k$ is a retraction functor from the category of all spaces onto the category of $k$-spaces. The weak Hausdorff property is stable under $k$. If $X$ and $Y$ are spaces, let $X \times Y=k\left(X \times{ }_{c} Y\right)$, where $\times{ }_{c}$ denotes the usual cartesian product. It is easy to check that $x$ is a product for the category of compactly generated spaces. One defines the product for systems analogously. As in [11] one forms mapping spaces by using first the compact-open topology and then applying the functor $k$.

No separation condition is required for the following proposition, whose proof is analogous to that of Theorem 4.4 in [11]. A proclusion is a surjective map whose range has the quotient topology.

2.2. Proposition. If $X$ and $Y$ are $k$-spaces and the maps $f: X \rightarrow X^{\prime}$ and $g: Y \rightarrow Y^{\prime}$ are proclusions, then $f \times g: X \times Y \rightarrow X^{\prime} \times Y^{\prime}$ is a proclusion.

2.3. Proposition. A $k$-space $X$ is a weak Hausdorff space if and only if the diagonal $\Delta_{X}$ is closed in $X \times X$.

Proof. Sufficiency: Suppose $\varphi$ is a map of a compact space $K$ into $X$. It suffices to show that $\varphi K$ is compactly closed. So let $\psi: L \rightarrow X$ be a map where $L$ is a compact space. Then $\psi^{-1} \varphi K=\operatorname{proj}_{2}(\varphi \times \psi)^{-1} \Delta_{X}$ is closed in $L$.

Necessity: We are to show that $\Delta_{X}$ is compactly closed in $X \times_{c} X$. So let $\varphi: K \rightarrow X \times_{c} X$ be a map where $K$ is compact. By 2.1, $A=\operatorname{proj}_{1} \varphi K \cup \operatorname{proj}_{2} \varphi K$ is a closed compact subspace of $X$. Now $\Delta_{A}$ is closed in $A \times{ }_{c} A$ and $\varphi K \subset A \times{ }_{c} A$, so $\varphi^{-1} \Delta_{X}=\varphi^{-1} \Delta_{A}$ is closed in $K$.

The following proposition is one of the reasons for the better closure properties of our category. Its proof is immediate from 2.2 and 2.3 , and from the fact that every quotient space of a $k$-space is a $k$-space.

2.4. Proposition. If $X$ is compactly generated and $p: X \rightarrow Y$ is a proclusion, then $Y$ is compactly generated if and only if $(p \times p)^{-1} \Delta_{Y}$ is closed in $X \times X$.

Let us call a map of pairs $h:(X, A) \rightarrow(Y, B)$ a relative homeomorphism provided $A$ is closed in $X, h$ maps $X$ proclusively onto $Y$, and $h$ maps $X-A$ bijectively to $Y-B$. It follows that $B$ is closed in $Y$, that $h$ maps $A$ proclusively onto $B$, and maps 
$X-A$ homeomorphically onto $Y-B$. It also follows that $h$ induces a homeomorphism of the adjunction space $X \cup_{h \mid A} B$ onto $Y$. Conversely, adjunction spaces give rise to relative homeomorphisms.

2.5. Proposition. If $h:(X, A) \rightarrow(Y, B)$ is a relative homeomorphism, where $X$ and $B$ are compactly generated, then $Y$ is compactly generated. In particular, $X / A$ is compactly generated.

Proof. $(h \times h)^{-1} \Delta_{Y}=\Delta_{X} \cup(h \times h)^{-1} \Delta_{B}$.

The following proposition is easy to establish.

2.6. Proposition. Suppose that a space $X$ is the union of an expanding sequence $\left(X_{0}, X_{1}, X_{2}, \ldots\right)$ of closed subspaces and $X$ has the topology of the union. If each $X_{n}$ is compactly generated, then so is $X$.

2.7. Convention. Throughout the rest of this paper we will assume that all spaces are compactly generated; correspondingly we usually omit the modifier "compactly generated". All products and mapping spaces are taken in this category (see above). The notions of topological group, group action, fiber bundle, et cetera, are modified in accordance with this notion of product.

The following lemma will be applied several times.

2.8. Lemma. Suppose given maps

$$
X \stackrel{p}{\longrightarrow} X^{\prime} \stackrel{i}{\longrightarrow} Y^{\prime} \stackrel{q}{\longleftarrow} Y,
$$

where $p$ is surjective, $i$ is injective, and $q$ is proclusive. Suppose $q^{-1} i X^{\prime}$ is the union of a finite collection $\left\{Y_{\alpha}\right\}$ of closed subsets of $Y$. Suppose there are maps $\rho_{\alpha}: Y_{\alpha} \rightarrow X$ making the following diagrams commutative:

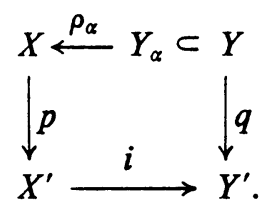

Then $i$ is a closed imbedding and $p$ is a proclusion.

Proof. We claim the following: If $F \subset X^{\prime}$ and $p^{-1} F$ is closed, then $i F$ is closed. For we have

$$
q^{-1} i F=\left(q^{-1} i F\right) \cap \bigcup_{\alpha} Y_{\alpha}=\bigcup_{\alpha}\left(q \mid Y_{\alpha}\right)^{-1} i F=\bigcup_{\alpha} \rho_{\alpha}^{-1} p^{-1} F,
$$

so that $q^{-1} i F$ is closed because it is a finite union of closed subsets of $Y$. Since $q$ is proclusive, $i F$ is then closed. This assertion, combined with the hypotheses that $p$ is a surjective map and $i$ is an injective map, clearly implies the conclusion.

We close this section by recalling a definition from [11]. A pair $(X, A)$ of spaces 
is called an NDR pair if there exist maps $h: X \times I \rightarrow X$ and $u: X \rightarrow I$ such that $A=u^{-1}(0), h(x, t)=x$ for $(x, t) \in(X \times 0) \cup(A \times I)$, and $h(x, 1) \in A$ for $x \in u^{-1}[0,1)$. Such a pair of maps $(h, u)$ is said to represent $(X, A)$ as an $N D R$ pair.

3. Based spaces. We denote all base points by *. A based space $X$ will be called nicely based if the inclusion $\{*\} \rightarrow X$ is a cofibration, or equivalently [11], $(X,\{*\})$ is an $N D R$ pair. We use the terminology based map for a base point preserving map, and based homotopy for a homotopy each level of which is a based map. If $X$ and $Y$ are based spaces, $[X, Y]$ denotes the set of all based homotopy classes of based maps $X \rightarrow Y$.

The smash product of based spaces $X$ and $Y$ is

$$
X \wedge Y=(X \times Y) /(X \times\{*\} \cup\{*\} \times Y) .
$$

It follows from 2.2 that the smash product is associative up to homeomorphism. We denote the image of $(x, y) \in X \times Y$ in $X \wedge Y$ by $x \wedge y$.

The unit interval $I$ and its boundary $S^{0}=\{0,1\}$ shall have base point 0 . We let $S^{1}=I / S^{0}$ and we denote the image of $t \in I$ in $S^{1}$ by $\bar{t}$. The $n$-sphere is $S^{n}=S^{1} \wedge \cdots$ $\wedge S^{1}$. We let $C X=I \wedge X, S X=S^{1} \wedge X$. The path space $P X$ of $X$ is the space of based maps $I \rightarrow X$, and the loop space $\Omega X$ is the subspace of $P X$ consisting of all $\alpha$ with $\alpha(1)=*$. For $\alpha \in P X$ and $s \in I$, we let $\alpha^{s} \in P X$ be given by $\alpha^{s}(t)=\alpha(s t)$.

If $f: X \rightarrow Y$ is a based map, the mapping track of $f$ is

$$
E_{f}=\{(x, \alpha) \in X \times P Y: f(x)=\alpha(1)\} .
$$

It is well known that if $f$ is a based (Hurewicz) fibration, then the canonical map $f^{-1}(*) \rightarrow E_{f}$, given by $x \mapsto(x, *)$, is a based homotopy equivalence.

3.1. LEMMA. If $f: X \rightarrow Y$ is a based map and $X$ is contractible, then the canonical map $j: \Omega Y \rightarrow E_{f}$, given by $j(\alpha)=(*, \alpha)$, is a based homotopy equivalence.

Proof. Let $h_{t}: X \rightarrow X$ be a contraction $\left(h_{0}=*, h_{1}=\mathrm{id}\right)$. We define a homotopy inverse $\psi: E_{f} \rightarrow \Omega Y$ for $j$ by

$$
\begin{aligned}
\psi(x, \alpha)(t) & =\alpha(2 t) & & \text { if } 0 \leqq t \leqq \frac{1}{2} \\
& =f h_{2-2 t}(x) & & \text { if } \frac{1}{2} \leqq t \leqq 1
\end{aligned}
$$

Now for any $\omega \in \Omega Y, \psi j(\omega)$ is given by $t \mapsto \omega(2 t)$ for $t \leqq \frac{1}{2}$ and $t \mapsto *$ for $t \geqq \frac{1}{2}$. So clearly $\psi \circ j \simeq$ id. On the other hand, define a homotopy $g_{t}: E_{f} \rightarrow E_{f}$ by $g_{t}(x, \alpha)$ $=\left(h_{t}(x), \psi(x, \alpha)^{(1-t / 2)}\right)$. One easily checks that $g_{0}=j \circ \psi$ and $g_{1}=\mathrm{id}$.

4. Numerable $G$-spaces. For a fixed topological group $G$, consider the category of (right) $G$-spaces and $G$-maps. We require of a $G$-space $E$ that the set

$$
\{(x, x \cdot g): x \in E, g \in G\}
$$

be closed in $E \times E$. By 2.4, this is equivalent to the condition that the orbit space $E / G$ remains compactly generated. It automatically holds when $G$ is compact. 
An equivariant partition of unity $\left(\pi_{j}\right)$ on a $G$-space $E$ is a system of $G$-maps $\pi_{j}: E \rightarrow I$ ( $I$ having the trivial $G$-action) such that (1) each point of $E$ is contained in an open $G$-subspace of $E$ intersecting only finitely many of the sets $\pi_{j}^{-1}(0,1]$, and (2) $\sum_{j} \pi_{j}(x)=1$ for each $x$ in $E$.

A $G$-space $E$ will be called numerable if there exist an equivariant partition of unity $\left(\pi_{j}\right)$ on $E$ and for each $j$ a $G$-map $\gamma_{j}: \pi_{j}^{-1}(0,1] \rightarrow G$. If $p: E \rightarrow E / G$ denotes the quotient map, then it is easy to see that $(E, p, E / G)$ is a locally trivial principal $G$-bundle which is numerable in the sense of Dold [3]. In particular, by [3] or [6], $p$ is a fibration - even more, for each choice of base point in $E, p$ is a based fibration.

4.1. Lemma. Suppose given $a G$-space $E$ and a sequence of $G$-maps $\rho_{n}: E \rightarrow I$, $n=0,1,2, \ldots$, such that $E$ is covered by the sets $\rho_{n}^{-1}(0,1]$. Then there exists an equivariant partition of unity $\left(\pi_{n}\right), n=0,1,2, \ldots$, on $E$ such that $\pi_{n}^{-1}(0,1] \subset \rho_{n}^{-1}(0,1]$ for each $n$.

Proof. First define $G$-maps $\tau_{n}: E \rightarrow I$ by

$$
\tau_{n}(x)=\max \left(0, \rho_{n}(x)-n \sum_{i=0}^{n-1} \rho_{i}(x)\right) .
$$

Clearly $\tau_{n}^{-1}(0,1] \subset \rho_{n}^{-1}(0,1]$. It can be checked that $\left(\tau_{n}^{-1}(0,1]\right)$ is an equivariantly locally finite open cover of $E$; compare with the argument on p. 237 of [3]. Then we let $\pi_{n}(x)=\tau_{n}(x) / \sum_{i=0}^{\infty} \tau_{i}(x)$.

The following theorem, which will be used in $\$ \$ 8$ and 9, can be viewed as a strengthening of Theorem 1.2 of Rothenberg and Steenrod [10].

4.2. TheOREM. Let $E$ be a $G$-space with action map $\varphi: E \times G \rightarrow E$. Suppose $E$ is the union (with the topology of the union) of a sequence of closed $G$-subspaces

$$
\varnothing=E_{-1} \subset E_{0} \subset E_{1} \subset \cdots \subset E_{n} \subset \ldots \subset E .
$$

Suppose that for each $n \geqq 0$ there exists a subspace $D_{n}$ of $E$ such that $\left(D_{n}, D_{n} \cap E_{n-1}\right)$ is an NDR pair and such that $\varphi$ defines a relative homeomorphism $\left(D_{n}, D_{n} \cap E_{n-1}\right)$ $\times G \rightarrow\left(E_{n}, E_{n-1}\right)$. Then $E$ is a numerable $G$-space.

Proof. Let us understand a pair of $G$-spaces to be a $G-N D R$ pair if it has a representation as an $N D R$ pair (see $\$ 2$ ) where both maps of the representation are $G$-maps ( $I$ having the trivial $G$-action). If we let $D_{n}$ above have the trivial $G$-action, then clearly $\left(D_{n}, D_{n} \cap E_{n-1}\right) \times G$ is a $G-N D R$ pair. Since $\varphi$ defines an equivariant relative homeomorphism from this pair to the pair $\left(E_{n}, E_{n-1}\right)$, it follows from the $G$-analog of Lemma 8.4 of [11] that $\left(E_{n}, E_{n-1}\right)$ is a $G-N D R$ pair. Then, by the $G$-analog of Theorem 9.4 of [11], $\left(E, E_{n}\right)$ is a $G-N D R$ pair for each $n \geqq-1$.

Let then $h_{n}: E \times I \rightarrow E$ and $u_{n}: E \rightarrow I$ be $G$-maps representing $\left(E, E_{n}\right)$ as an $N D R$ pair. We are going to apply Lemma 4 .1. For each $n \geqq 0$ define a $G$-map $\rho_{n}: E \rightarrow I$ by

$$
\rho_{n}(x)=\left(1-u_{n}(x)\right) \cdot u_{n-1}\left(h_{n}(x, 1)\right) .
$$


It is easy to check that

$$
E_{n}-E_{n-1} \subset \rho_{n}^{-1}(0,1] \subset h_{n}(\cdot, 1)^{-1}\left(E_{n}-E_{n-1}\right) .
$$

Because of the first inclusion, the sets $\rho_{n}^{-1}(0,1]$ obviously cover $E$. We can define a $G$-map $\gamma_{n}: \rho_{n}^{-1}(0,1] \rightarrow G$ as the composition of the following three $G$-maps:

$$
\rho_{n}^{-1}(0,1] \rightarrow E_{n}-E_{n-1} \rightarrow\left(D_{n}-\left(D_{n} \cap E_{n-1}\right)\right) \times G \rightarrow G .
$$

The first is the restriction of $h_{n}(\cdot, 1)$, which exists because of (4.3). The second is defined by the inverse of the relative homeomorphism defined by $\varphi$. The third is the projection. Now if we apply 4.1 , we get an equivariant partition of unity $\left(\pi_{n}\right)$ on $E$ such that $\pi_{n}^{-1}(0,1] \subset \rho_{n}^{-1}(0,1]$. The $G$-maps $\gamma_{n}$ restrict to $G$-maps $\pi_{n}^{-1}(0,1] \rightarrow G$, and the proof is complete.

5. The monoids $B(G, X)$. Let $G$ be a monoid-a set with an associative multiplication having a two-sided unit $e$. Let $X$ be a based set. We let $B(G, X)$ denote the set of all functions $u: X \rightarrow G$ such that $u(*)=e$ and such that $u(x)=e$ for all but finitely many $x$ in $X . B(G, X)$ is again a monoid under pointwise multiplication of functions. Let this operation be denoted by $\oplus$. (Thus $\left(u \oplus u^{\prime}\right)(x)=u(x) \cdot u^{\prime}(x)$.) If $G$ is abelian, then so is $B(G, X)$. If $G$ is a group, then so is $B(G, X)$.

If $g \in G$ and $x \in X-\{*\}$, let $g x$ denote the element of $B(G, X)$ whose value at $x$ is $g$ and whose value elsewhere is $e$. We agree that $g *$ is the unit of $B(G, X)$, the function whose value everywhere is $e$.

For each integer $n \geqq 0$, let $B_{n}(G, X)$ be the set of members of $B(G, X)$ whose value is $e$ on at most $n$ elements of $X$. Thus $B_{0}(G, X)=\{e\}$ and for $n \geqq 1, B_{n}(G, X)$ consists of those elements expressible in the form $g_{1} x_{1} \oplus \cdots \oplus g_{n} x_{n}, g_{i} \in G, x_{i} \in X$. We have $B(G, X)$ as the union of an increasing sequence of subsets

$$
B_{0}(G, X) \subset B_{1}(G, X) \subset \ldots \subset B_{n}(G, X) \subset \ldots \subset B(G, X) .
$$

5.2. Proposition. If $\varphi: G \rightarrow G^{\prime}$ is a morphism of monoids and $\psi: X \rightarrow X^{\prime}$ is a morphism of based sets, then there exists a unique morphism of monoids

satisfying

$$
B(\varphi, \psi): B(G, X) \rightarrow B\left(G^{\prime}, X^{\prime}\right)
$$

$$
B(\varphi, \psi)\left(g_{1} x_{1} \oplus \cdots \oplus g_{n} x_{n}\right)=\left(\varphi g_{1}\right)\left(\psi x_{1}\right) \oplus \cdots \oplus\left(\varphi g_{n}\right)\left(\psi x_{n}\right)
$$

for all $g_{i} \in G, x_{i} \in X$, provided at least one of the following conditions holds: (a) $G^{\prime}$ is abelian, (b) $\psi$ is injective on $X-\psi^{-1}(*)$.

Proof. If (a) or (b) holds, then we can define $B(\varphi, \psi)$ as follows. If $u \in B(G, X)$, let $B(\varphi, \psi)(u)$ be the based function $X^{\prime} \rightarrow G^{\prime}$ whose value at each $x^{\prime} \in X^{\prime}-\{*\}$ is the product in $G^{\prime}$ of the finite set $\left\{\varphi(u(x)): x \in \psi^{-1}\left(x^{\prime}\right), u(x) \neq e\right\}$, where it is understood that the product of the empty set is $e$. The product of this set is well defined if $G^{\prime}$ is abelian, and it is well defined under condition (b) because then the set has at most one element. It is easy to check that $B(\varphi, \psi)$ is a homomorphism 
and that $B(\varphi, \psi)(g x)=(\varphi g)(\psi x)$ for arbitrary $g \in G, x \in X$. Hence (5.3) holds. Uniqueness is obvious.

We shall denote $B(\varphi, i d)$ simply by $\varphi_{*}$ and denote $B(i d, \psi)$ by $\psi_{*}$ whenever it is defined. From this proposition one can note the various ways in which the construction $B(\cdot, \cdot)$ can be restricted to be a funcior, covariant in both variables.

6. The abelian topological monoids $B(G, X)$. In this section we suppose $G$ is an abelian topological monoid (written additively) and $X$ is a based space. We are going to put a topology on $B(G, X)$ that will make it again an abelian topological monoid. We recall Convention 2.7; we are working in the category of compactly generated spaces. We shall want to verify that $B(G, X)$ remains in that category.

For each $n \geqq 0$ we make $B_{n}(G, X)$ a space by giving it the quotient topology from the following map $\mu_{n}$ whose domain is the $n$-fold product $(G \times X)^{n}$ :

$$
\begin{gathered}
(G \times X)^{n} \stackrel{\mu_{n}}{\longrightarrow} B_{n}(G, X), \\
\left(\left(g_{1}, x_{1}\right), \ldots,\left(g_{n}, x_{n}\right)\right) \mapsto g_{1} x_{1} \oplus \cdots \oplus g_{n} x_{n} .
\end{gathered}
$$

We understand that $\mu_{0}(\varnothing)=0$.

6.2. LemmA. For each $n>0, B_{n-1}(G, X)$ is a closed subspace of $B_{n}(G, X)$.

6.3. Definition. If $G$ is an abelian topological monoid and $X$ is a based space, then $B(G, X)$ shall have the topology of the union of the expanding sequence of spaces $B_{n}(G, X)$, where $B_{n}(G, X)$ has the quotient topology from (6.1).

Proof of 6.2. The inclusion map $i: B_{n-1}(G, X) \rightarrow B_{n}(G, X)$ is continuous because it can be covered by the map $(G \times X)^{n-1} \rightarrow(G \times X)^{n}$ that tacks on the coordinate $(0, *)$. To see that $i$ is a closed map we apply 2.8 to the maps

$$
(G \times X)^{n-1} \stackrel{\mu_{n-1}}{\longrightarrow} B_{n-1}(G, X) \stackrel{i}{\longrightarrow} B_{n}(G, X) \stackrel{\mu_{n}}{\longleftarrow}(G \times X)^{n} .
$$

For $1 \leqq j \leqq n$ and $1 \leqq k<l \leqq n$, define subsets of $(G \times X)^{n}$ as follows:

$$
\begin{aligned}
Y_{j} & =\left\{\left(\left(g_{1}, x_{1}\right), \ldots,\left(g_{n}, x_{n}\right)\right): g_{j}=0 \text { or } x_{j}=*\right\}, \\
Y_{k l} & =\left\{\left(\left(g_{1}, x_{1}\right), \ldots,\left(g_{n}, x_{n}\right)\right): x_{k}=x_{l}\right\}
\end{aligned}
$$

It follows from the weak Hausdorff property of $X$ and $G$ and from 2.3 that $Y_{j}$ and $Y_{k l}$ are closed in $(G \times X)^{n}$. It is easy to see that $\mu_{n}^{-1} B_{n-1}(G, X)$ is the union of the $Y_{j}$ and the $Y_{k l}$. Let $\rho_{j}: Y_{j} \rightarrow(G \times X)^{n-1}$ be the map that omits the $j$ th coordinate $\left(g_{j}, x_{j}\right)$. Let $\rho_{k l}: Y_{k l} \rightarrow(G \times X)^{n-1}$ be the map that replaces the $k$ th coordinate $\left(g_{k}, x_{k}\right)$ by $\left(g_{k}+g_{l}, x_{k}\right)$ and omits the lth coordinate. Then it is easy to check that the hypotheses of 2.8 are satisfied.

For the purposes of the next lemma and of Theorem 9.18 let us introduce the 
following notation. Let $S_{n}$ be the group of permutations of $\{1,2, \ldots, n\}$. For any space $Y$ define a right action

$$
Y^{n} \times S_{n} \rightarrow Y^{n}, \quad\left(y_{1}, \ldots, y_{n}\right) \cdot \alpha=\left(y_{\alpha(1)}, \ldots, y_{\alpha(n)}\right) .
$$

The orbit space $S P^{n} Y=Y^{n} / S_{n}$ (the $n$-fold symmetric product of $Y$ ) is still compactly generated by the first paragraph of $\S 4$.

6.5. LEMMA. $B(G, X)$ is compactly generated.

Proof. By 6.3 and 2.6 it suffices to show that each $B_{n}(G, X)$ is compactly generated. The case $n=0$ is trivial. Suppose $n>0$ and suppose, inductively, that $B_{n-1}(G, X)$ is compactly generated. Since $G$ is abelian, the proclusion $\mu_{n}$ factors through the proclusion $(G \times X)^{n} \rightarrow S P^{n}(G \times X)$, giving a proclusion

$$
\bar{\mu}_{n}: S P^{n}(G \times X) \rightarrow B_{n}(G, X) .
$$

It is easy to check that $\bar{\mu}_{n}$ is injective on the complement of $\bar{\mu}_{n}^{-1} B_{n-1}(G, X)$. Since $B_{n-1}(G, X)$ is closed, then, $\bar{\mu}_{n}$ defines a relative homeomorphism

$$
\left(S P^{n}(G \times X), \bar{\mu}_{n}^{-1} B_{n-1}(G, X)\right) \rightarrow\left(B_{n}(G, X), B_{n-1}(G, X)\right) .
$$

Hence by $2.5, B_{n}(G, X)$ is compactly generated.

6.6. Proposition. If $G$ is an abelian topological monoid and $X$ is a based space, then $B(G, X)$ is an abelian topological monoid. If further $G$ is an abelian topological group, then so is $B(G, X)$.

Proof. To verify continuity of the addition in $B(G, X)$ (defined in $\S 5$ ) it suffices by $6.3,6.5$, and Theorem 10.3 of [11], to show that its restriction to $B_{m}(G, X)$ $\times B_{n}(G, X)$ is continuous for all $m, n \geqq 0$. But this restriction can be put into a commutative diagram

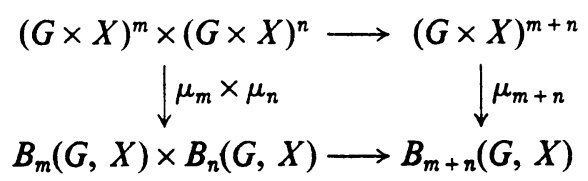

where the upper map is the obvious homeomorphism. Since $\mu_{m} \times \mu_{n}$ is a proclusion by 2.2 , the result follows. Similarly if $G$ has a continuous inversion, then the inversion in $B(G, X)$ is seen to be continuous by looking at the commutative diagrams

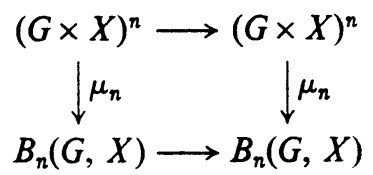

where the upper map sends $\left(\left(g_{1}, x_{1}\right), \ldots,\left(g_{n}, x_{n}\right)\right)$ to $\left(\left(-g_{1}, x_{1}\right), \ldots,\left(-g_{n}, x_{n}\right)\right)$. 
6.7. Proposition. Suppose $\varphi: G \rightarrow G^{\prime}$ is a morphism of abelian topological monoids and $\psi: X \rightarrow X^{\prime}$ is a based map. Let

$$
B(\varphi, \psi): B(G, X) \rightarrow B\left(G^{\prime}, X^{\prime}\right)
$$

be defined as in 5.2. Then the following three statements hold. (a) $B(\varphi, \psi)$ is a morphism of abelian topological monoids. (b) If $\varphi$ and $\psi$ are proclusions, then so is $B(\varphi, \psi)$. (c) If $\varphi$ and $\psi$ are injective closed maps, then so is $B(\varphi, \psi)$.

Proof. (a) By 5.2 we need only show $B(\varphi, \psi)$ is continuous. But for each $n \geqq 0$ we have a commutative diagram

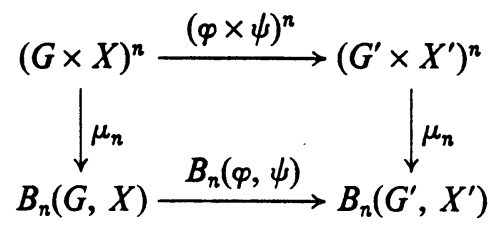

where $B_{n}(\varphi, \psi)$, the restriction of $B(\varphi, \psi)$, must therefore be continuous. Thus the result follows from 6.3. (b) By 6.3 it suffices to show that each $B_{n}(\varphi, \psi)$ is a proclusion. But this is seen by looking at (6.8), where $(\varphi \times \psi)^{n}$ must be a proclusion by 2.2. (c) It suffices to show that each $B_{n}(\varphi, \psi)$ is a closed map. We do this by applying 2.8 to the maps

$$
(G \times X)^{n} \stackrel{\mu_{n}}{\longrightarrow} B_{n}(G, X) \stackrel{B_{n}(\varphi, \psi)}{\longrightarrow} B_{n}\left(G^{\prime}, X^{\prime}\right) \stackrel{\mu_{n}}{\longleftarrow}\left(G^{\prime} \times X^{\prime}\right)^{n} .
$$

For each collection $\alpha$ of pairwise disjoint subsets of $\{1, \ldots, n\}$, let $Y_{\alpha}$ consist of those elements $\left(\left(g_{1}^{\prime}, x_{1}^{\prime}\right), \ldots,\left(g_{n}^{\prime}, x_{n}^{\prime}\right)\right)$ of $\left(G^{\prime} \times X^{\prime}\right)^{n}$ satisfying the following two conditions. (1) For each $J \in \alpha, x_{j}^{\prime}=x_{k}^{\prime}$ for all $j, k \in J$, and $\sum_{j \in J} g_{j}^{\prime}=0$. (2) If

$$
j \in\{1, \ldots, n\}-\bigcup \alpha,
$$

then $g_{j}^{\prime} \in \varphi G$ and $x_{j}^{\prime} \in \psi X$. Then we let $\rho_{\alpha}: Y_{\alpha} \rightarrow(G \times X)^{n}$ be the map which acts on $\left(\left(g_{1}^{\prime}, x_{1}^{\prime}\right), \ldots,\left(g_{n}^{\prime}, x_{n}^{\prime}\right)\right)$ by replacing $\left(g_{j}^{\prime}, x_{j}^{\prime}\right)$ by $(0, *)$ if $j \in \bigcup_{\alpha}$ and by replacing $\left(g_{j}^{\prime}, x_{j}^{\prime}\right)$ by $\left(\varphi^{-1} g_{j}^{\prime}, \psi^{-1} x_{j}^{\prime}\right)$ otherwise. One can check that the hypotheses of 2.8 are satisfied.

6.9. Corollary. If $\mathscr{A}$ and $\mathscr{B}$ are the categories of abelian topological monoids and of based spaces, respectively, then $B(\cdot, \cdot)$ is a bifunctor $\mathscr{A} \times \mathscr{B} \rightarrow \mathscr{A}$, covariant in both variables.

6.10. Proposition. If $\psi_{t}: X \rightarrow X^{\prime}$ is a based homotopy and $G$ is an abelian topological monoid, then $\left(\psi_{t}\right)_{*}: B(G, X) \rightarrow B\left(G, X^{\prime}\right)$ is a homotopy.

The proof is similar to that of $6.7(\mathrm{a})$.

6.11. Corollary. If $X$ and $X^{\prime}$ have the same based homotopy type, then so do $B(G, X)$ and $B\left(G, X^{\prime}\right)$. In particular, if $X$ is contractible, then so is $B(G, X)$. 
For the last proposition in this section it is convenient to exhibit $B(G, X)$ directly as a quotient space. For any space $Y$, let $M(Y)$ be the topological sum of the spaces $Y^{n}, n=0,1,2, \ldots$ Let

$$
M(G \times X) \stackrel{\mu}{\rightarrow} B(G, X)
$$

be the map whose restriction to $(G \times X)^{n}$ is $\mu_{n}$ followed by the inclusion $B_{n}(G, X) \rightarrow$ $B(G, X)$. It is trivial to check that $\mu$ is a proclusion.

6.13. Proposition. If $G$ is an abelian topological monoid and $X$ and $Y$ are based spaces, then $B(G, X \wedge Y)$ and $B(B(G, X), Y)$ are isomorphic as abelian topological monoids.

Proof. Consider the following diagram, where all vertical arrows represent the obvious proclusions (three of them involving (6.12)):

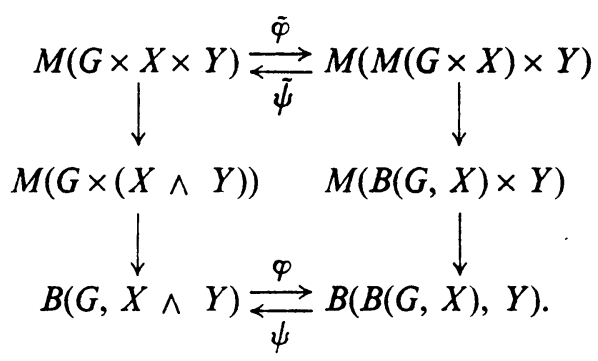

Here $\varphi$ is the obvious algebraic morphism well defined by $\varphi\left(\sum_{i} g_{i}\left(x_{i} \wedge y_{i}\right)\right)=$ $\sum_{i}\left(g_{i} x_{i}\right) y_{i}$, and $\psi$ is defined by $\psi\left(\sum_{j}\left(\sum_{i} g_{i j} x_{i j}\right) y_{j}\right)=\sum_{i, j} g_{i j}\left(x_{i j} \wedge y_{j}\right)$. One checks that $\varphi$ and $\psi$ are mutual inverses. They are "covered" in the diagram by $\tilde{\varphi}$ and $\psi$, respectively, defined by

and

$$
\tilde{\varphi}\left(\left(g_{1}, x_{1}, y_{1}\right), \ldots,\left(g_{n}, x_{n}, y_{n}\right)\right)=\left(\left(\left(g_{1}, x_{1}\right), y_{1}\right), \ldots,\left(\left(g_{n}, x_{n}\right), y_{n}\right)\right)
$$

$$
\begin{aligned}
& \left.\tilde{\psi}\left(\left(\left(g_{11}, x_{11}\right), \ldots,\left(g_{m_{1} 1}, x_{m_{1} 1}\right)\right), y_{1}\right), \ldots,\left(\left(\left(g_{1 n}, x_{1 n}\right), \ldots,\left(g_{m_{n} n}, x_{m_{n} n}\right)\right), y_{n}\right)\right) \\
& \quad=\left(\left(g_{11}, x_{11}, y_{1}\right), \ldots,\left(g_{m_{1} 1}, x_{m_{1} 1}, y_{1}\right), \ldots,\left(g_{1 n}, x_{1 n}, y_{n}\right), \ldots,\left(g_{m_{n} n}, x_{m_{n} n}, y_{n}\right)\right) .
\end{aligned}
$$

Hence $\varphi$ and $\psi$ are continuous, and the proof is complete.

7. CW structure on $B(G, X)$ for $G$ discrete abelian and $X$ triangulable. Throughout this section let $G$ be a discrete abelian monoid and let $X$ be a based simplicial complex, with the weak topology. Let us order the vertices of $X$ so that we can handle products. We will show that these data determine a $\mathrm{CW}$ structure on $B(G, X)$. The proof is similar to that of an analogous theorem in [4].

In the following, simplex will mean open simplex and cell will mean open cell. Let $\Delta^{r}$ denote the standard closed $r$-simplex $\left\{\left(\lambda_{0}, \ldots, \lambda_{r}\right): \lambda_{i} \geqq 0, \sum_{i} \lambda_{i}=1\right\}$.

It is convenient to identify $G^{n} \times X^{n}$ with $(G \times X)^{n}$ in $M(G \times X)$ under the obvious homeomorphism. Give each $n$-fold product $X^{n}$ the standard simplicial structure of 
a product [5, pp. 66-70]. Since $G$ is discrete, $G^{n} \times X^{n}$ is then a simplicial complex with simplexes of the form $g \times \sigma$, where $g \in G^{n}$ and $\sigma$ is a simplex of $X^{n}$. Hence $M(G \times X)$ has a simplicial structure. The idea is to carry this over to a $\mathrm{CW}$ structure on $B(G, X)$ by the proclusion $\mu$ (see (6.12)).

The $r$-simplexes of the $n$-fold product $X^{n}$ are in bijective correspondence with $(r+1) \times n$ matrices

$$
J=\left(\begin{array}{cccc}
v_{01} & v_{02} & \cdots & v_{0 n} \\
v_{11} & v_{12} & \cdots & v_{1 n} \\
\vdots & \vdots & & \vdots \\
v_{r 1} & v_{r 2} & \cdots & v_{r n}
\end{array}\right)
$$

of vertices of $X$ satisfying the two conditions:

(A) the rows of $J$ are distinct;

(B) for each $j=1, \ldots, n$, the vertices in the $j$ th column satisfy $v_{0 j} \leqq \cdots \leqq v_{r j}$ and span a simplex of $X$.

If $\sigma_{J}$ denotes the $r$-simplex of $X^{n}$ corresponding to $J$, then the vertices of $\sigma_{J}$ are just the rows of $J$ and the linear structure of $\bar{\sigma}_{J}$ is given by the characteristic map $\Delta^{r} \rightarrow \bar{\sigma}_{J}$ sending $\left(\lambda_{0}, \ldots, \lambda_{r}\right)$ to $\left(\sum_{i} \lambda_{i} v_{i 1}, \ldots, \sum_{i} \lambda_{i} v_{i n}\right)$. Thus the $r$-simplexes of $G^{n} \times X^{n}$ are of the form $g \times \sigma_{J}$ where $g \in G^{n}$ and $J$ is as above.

Call a matrix $J$ of the form (7.1) satisfying (A) and (B) nondegenerate if it also satisfies the following two conditions.:

(C) the columns of $J$ are distinct;

(D) no column of $J$ has all entries equal to the basepoint of $X$.

7.2. Lemma. Let $J=\left(v_{i j}\right)$ and $J^{\prime}=\left(v_{i j}^{\prime}\right)$ be nondegenerate matrices of size $(r+1) \times n$ and $\left(r^{\prime}+1\right) \times n^{\prime}$, respectively, and let $g \in(G-0)^{n}, g^{\prime} \in(G-0)^{n^{\prime}}$. Suppose given points $\lambda \in$ int $\Delta^{r}$ and $\lambda^{\prime} \in$ int $\Delta^{r^{\prime}}$ such that

$$
g_{1}\left(\sum_{i} \lambda_{i} v_{i 1}\right) \oplus \cdots \oplus g_{n}\left(\sum_{i} \lambda_{i} v_{i n}\right)=g_{i}^{\prime}\left(\sum_{i} \lambda_{i}^{\prime} v_{i 1}^{\prime}\right) \oplus \cdots \oplus g_{n^{\prime}}^{\prime}\left(\sum_{i} \lambda_{i}^{\prime} v_{i n^{\prime}}^{\prime}\right)
$$

in $B(G, X)$. Then $\lambda=\lambda^{\prime}$ and $\mu\left(g \times \sigma_{J}\right)=\mu\left(g^{\prime} \times \sigma_{J^{\prime}}\right)$.

Proof. From conditions (B) and (C) and the fact that $\lambda \in$ int $\Delta^{r}$, one easily sees that $\sum_{i} \lambda_{i} v_{i 1}, \ldots, \sum_{i} \lambda_{i} v_{i n}$ represent $n$ distinct points of $X$. From (B), (D), and $\lambda \in$ int $\Delta^{r}$, it follows that none of these $n$ points is the base point of $X$. A similar conclusion is made about the primed case. Since none of the $g_{i}$ or $g_{i}^{\prime}$ are 0 , it follows then from the equation of the hypothesis that $n=n^{\prime}$ and that there is a permutation $\varphi$ of $\{1, \ldots, n\}$ such that $\left(g_{j}^{\prime}, \sum_{i} \lambda_{i}^{\prime} c_{i j}^{\prime}\right)=\left(g_{\varphi(j)}, \sum_{i} \lambda_{i} v_{i \varphi(j)}\right)$ for each $j=1, \ldots, n$. In particular,

$$
\left(\sum_{i} \lambda_{i}^{\prime} v_{i 1}^{\prime}, \ldots, \sum_{i} \lambda_{i}^{\prime} v_{i n}^{\prime}\right)=\left(\sum_{i} \lambda_{i} v_{i \varphi(1)}, \ldots, \sum_{i} \lambda_{i} v_{i \varphi(n)}\right)
$$


in $X^{n}$, which means that the simplexes of $X^{n}$ corresponding to the matrices $\left(v_{i j}^{\prime}\right)$ and $\left(v_{i \varphi(j)}\right)$ have a point in common. Hence these simplexes are the same and their matrices are the same: $\left(v_{i j}^{\prime}\right)=\left(v_{i \varphi(j)}\right)$. It follows then from equation (7.3) that $\lambda=\lambda^{\prime}$. Finally, note that for any $\alpha \in$ int $\Delta^{r}$, we have now the equations in $B(G, X)$ :

$$
\begin{aligned}
g_{1}\left(\sum_{i} \alpha_{i} v_{i 1}\right) \oplus \cdots \oplus g_{n}\left(\sum_{i} \alpha_{i} v_{i n}\right) & =g_{\varphi(1)}\left(\sum_{i} \alpha_{i} v_{i \varphi(1)}\right) \oplus \cdots \oplus g_{\varphi(n)}\left(\sum_{i} \alpha_{i} v_{i \varphi(n)}\right) \\
& =g_{i}^{\prime}\left(\sum_{i} \alpha_{i} v_{i 1}^{\prime}\right) \oplus \cdots \oplus g_{n}^{\prime}\left(\sum_{i} \alpha_{i} v_{i n}^{\prime}\right) .
\end{aligned}
$$

This implies that $\mu\left(g \times \sigma_{J}\right)=\mu\left(g^{\prime} \times \sigma_{J}\right)$, and the proof is complete.

Let us call a simplex $g \times \sigma_{J}$ of $M(G \times X)$ nondegenerate if $g \in(G-0)^{n}$ and $J$ is nondegenerate. We wish to apply to our situation the following general lemma.

7.4. Lemma. Let $\mu: M \rightarrow B$ be a proclusion, where $M$ is a $C W$-complex and $B$ is a topological space. Suppose there exists a collection of cells of $M$ called the nondegenerate cells satisfying the following three conditions.

(i) $\mu$ is injective on each nondegenerate cell.

(ii) The images under $\mu$ of any two nondegenerate cells are either disjoint or equal.

(iii) Given any cell $e$ of $M$, there exists a nondegenerate cell $e^{\prime}$ of no greater dimension and a map $f$ of $(\bar{e}, e)$ onto $\left(\bar{e}^{\prime}, e^{\prime}\right)$ such that $\mu f(x)=\mu(x)$ for all $x$ in $\bar{e}$.

Then the sets $\mu(e)$, where e varies over the nondegenerate cells of $M$, form a $C W$ decomposition of $B$. For a characteristic map for $\mu(e)$ we can take the composite of $\mu$ with a characteristic map for $e$.

The proof of this lemma is an easy generalization of the proof of Milnor's Lemma 3.2 in [8], and will therefore be omitted.

Condition (i) of this lemma is verified immediately for our situation (for the proclusion $\mu: M(G \times X) \rightarrow B(G, X))$ by applying Lemma 7.2 with $(g, J)=\left(g^{\prime}, J^{\prime}\right)$ and taking the first part of the conclusion (that $\lambda=\lambda^{\prime}$ ). Condition (ii) follows immediately from Lemma 7.2 with the second part of the conclusion.

To verify condition (iii), let $g \times \sigma_{J}$ be an arbitrary simplex of $G^{n} \times X^{n}$, where $J$ is a matrix of the form (7.1) satisfying (A) and (B). If $g \times \sigma_{J}$ is already nondegenerate, we just take $f$ to be the identity on $g \times \sigma_{J}$. Otherwise we obtain from $g \times \sigma_{J}$ a "contracted" nondegenerate simplex by applying successively to the pair $(g, J)$ the following four operations, each of which is defined for any such pair $(g, J)$.

(1) Omit from $J$ any column, all of whose entries are the basepoint of $X$, and omit from $g$ the corresponding $g_{j}$ 's.

(2) "Collecting $g$ coefficients." If the first column of $J$ coincides with some later columns, then omit these later columns, and omit the corresponding entries of $g$. Replace however $g_{1}$ by its sum with these later entries. Then look at the new second column and continue the process.

(3) Omit any zero entries of $g$ and omit the corresponding columns of $J$. 
(4) "Omitting superfluous rows." If the first row of $J$ coincides with some later rows, omit these later rows. Then look at the new second row and continue the process. This operation has no effect on $g$.

Let $\left(g^{\prime}, J^{\prime}\right)$ be the result of applying the first three operations to our original $(g, J), J^{\prime}=\left(v_{i j}^{\prime}\right)$ being of size $(r+1) \times n^{\prime}, n^{\prime} \leqq n$. Let $\left(g^{\prime}, J^{\prime \prime}\right)$ be the result of applying operation (4) to $\left(g^{\prime}, J^{\prime}\right), J^{\prime \prime}$ being of size $\left(r^{\prime}+1\right) \times n^{\prime}, r^{\prime} \leqq r$. Then it is clear that $g^{\prime} \times \sigma_{J "}$ is a nondegenerate simplex. We define a linear map $f: g \times \bar{\sigma}_{J} \rightarrow g^{\prime} \times \bar{\sigma}_{J^{\prime \prime}}$ by the equation

$$
f\left(g,\left(\sum_{i} \lambda_{i} v_{i 1}, \ldots, \sum_{i} \lambda_{i} v_{i n}\right)\right)=\left(g^{\prime},\left(\sum_{i} \lambda_{i} v_{i 1}^{\prime}, \ldots, \sum_{i} \lambda_{i} v_{i n^{\prime}}^{\prime}\right)\right)
$$

for $\lambda \in \Delta^{r}$. Clearly $f\left(g \times \sigma_{J}\right)=g^{\prime} \times \sigma_{J^{\prime \prime}}$, and from the way $\left(g^{\prime}, J^{\prime \prime}\right)$ was obtained, $\mu f(u)=\mu(u)$ for all $u \in g \times \bar{\sigma}_{J}$.

Thus we can apply Lemma 7.4, and we know that the sets $\mu\left(g \times \sigma_{J}\right)$, where $g \times \sigma_{J}$ varies over the nondegenerate simplexes of $M(G \times X)$, form a $\mathrm{CW}$ decomposition of $B(G, X)$. For each such cell $\mu\left(g \times \sigma_{J}\right)$ with $J=\left(v_{i j}\right)$ of size $(r+1) \times n$, we can take as characteristic map the map

$$
\varphi: \Delta^{r} \rightarrow \overline{\mu\left(g \times \sigma_{J}\right)}
$$

given by

$$
\varphi\left(\lambda_{0}, \ldots, \lambda_{r}\right)=g_{1}\left(\sum_{i} \lambda_{i} v_{i 1}\right) \oplus \cdots \oplus g_{n}\left(\sum_{i} \lambda_{i} v_{i n}\right)
$$

7.6. REMARK. If $G$ is a countable discrete monoid and $X$ is a countable simplicial complex, then $B(G, X)$ is clearly a countable $C W$ complex. Hence the product $B(G, X) \times B(G, X)$ is the same as the cartesian product. Therefore in this case $B(G, X)$ is a topological monoid in the usual sense.

8. The principal bundle $B(G, A) \rightarrow B(G, X) \rightarrow B(F, X \mid A)$. In the interests of (a) possible generalizations of the main theorem (8.8), and (b) the relations of $\S 9$ to the present section, we are going to start off more generally than we will end up. Suppose $G$ is an abelian topological monoid and $(X, A)$ is a closed pair of based spaces. Then if $i: A \rightarrow X$ is the inclusion and $p: X \rightarrow X / A$ is the proclusion, we have the morphisms

$$
B(G, A) \stackrel{i_{*}}{\longrightarrow} B(G, X) \stackrel{p_{*}}{\longrightarrow} B(G, X / A) .
$$

By $6.7, i_{*}$ is a closed imbedding and $p_{*}$ is a proclusion.

We can use $i_{*}$ and the operation in $B(G, X)$ to define an action of $B(G, A)$ on $B(G, X)$ :

$$
B(G, X) \times B(G, A) \stackrel{\varphi}{\longrightarrow} B(G, X), \quad \varphi(u, v)=u \oplus i_{*} v .
$$

8.3. Lemma. $p_{*}$ induces $B(G, X) / B(G, A) \approx B(G, X / A)$. 
Proof. For each $u \in B(G, X)$, let $[u] \in B(G, X) / B(G, A)$ denote the orbit of $u$. Since $p_{*}$ is a proclusion, it suffices to show that $[u]=\left[u^{\prime}\right]$ if and only if $p_{*} u=p_{*} u^{\prime}$. The only if part is implied by $p_{*} i_{*}=0$. Conversely, suppose $p_{*} u=p_{*} u^{\prime}$. Write $u=u_{0} \oplus i_{*} v$ where $u_{0}(x)=0$ for $x \in A$ and $v \in B(G, A)$. Write $u^{\prime}=u_{0}^{\prime} \oplus i_{*} v^{\prime}$ similarly. Then $p_{*} u_{0}=p_{*} u=p_{*} u^{\prime}=p_{*} u_{0}^{\prime}$. Hence $u_{0}=u_{0}^{\prime}$, and $[u]=\left[u_{0}\right]=\left[u_{0}^{\prime}\right]=\left[u^{\prime}\right]$.

Now for each $n \geqq 0$ define a $B(G, A)$-invariant, closed subspace $E_{n}$ of $B(G, X)$ by

$$
E_{n}=p_{*}^{-1} B_{n}(G, X / A)=B_{n}(G, X) \oplus i_{*} B(G, A) .
$$

We also write $E_{-1}=\varnothing$. Note that $B(G, X)$ has the topology of the union of the $E_{n}$ since it has the topology of the union of the subspaces $B_{n}(G, X)$.

8.5. Lemma. An element $g_{1} x_{1} \oplus \cdots \oplus g_{n} x_{n}$ of $B_{n}(G, X)$ is also in $E_{n-1}$ if and only if (a) some $g_{j}=0$, or (b) some $x_{j} \in A$, or (c) $x_{j}=x_{k}$ for some $j \neq k$.

\subsection{LEMMA. For each $n \geqq 0$ the action map $\varphi$ defines a relative homeomorphism}

$$
\left(B_{n}(G, X), B_{n}(G, X) \cap E_{n-1}\right) \times B(G, A) \rightarrow\left(E_{n}, E_{n-1}\right) .
$$

Proof. One can use 8.5 to check that $\varphi$ defines a map of pairs as indicated, and that $\varphi$ maps $\left(B_{n}(G, X)-B_{n}(G, X) \cap E_{n-1}\right) \times B(G, A)$ bijectively to $E_{n}-E_{n-1}$. To show that $\varphi$ maps $B_{n}(G, X) \times B(G, A)$ proclusively to $E_{n}$, it suffices to show that $\varphi$ maps $B_{n}(G, X) \times B_{m}(G, A)$ proclusively to $E_{n} \cap B_{n+m}(G, X)$ for each $m \geqq 0$. For this, it suffices to show that the first map in the following diagram is a proclusion:

$$
\begin{aligned}
(G \times X)^{n} \times(G \times A)^{m} \stackrel{\varphi \circ\left(\mu_{n} \times \mu_{m}\right)}{\longrightarrow} E_{n} \cap B_{n+m}(G, X) \subset B_{n+m}(G, X) \\
\stackrel{\mu_{n+m}}{\longleftarrow}(G \times X)^{n+m} .
\end{aligned}
$$

But 2.8 can be applied to these maps. We omit the details, which are similar to those of $6.7(c)$.

8.7. LEMMA. If $G$ is discrete abelian monoid and $(X, A)$ is a based triangulable pair, then for each $n \geqq 0,\left(B_{n}(G, X), B_{n}(G, X) \cap E_{n-1}\right)$ is an NDR pair.

Proof. By the preceding section, $B(G, X)$ has a $\mathrm{CW}$ structure. It is easy to see that $B_{n}(G, X)$ and $B_{n}(G, X) \cap E_{n-1}$ (refer to 8.5) are subcomplexes. Hence the result.

If, in addition to the hypotheses of $8.7, G$ is a group, then by 8.6 and 8.7 we can apply Theorem 4.2 to $B(G, X)$. This, in combination with 8.3 , gives:

8.8. THEOREM. If $G$ is a discrete abelian group and $(X, A)$ is a based triangulable pair, then $\left(B(G, X), p_{*}, B(G, X / A)\right)$ is a numerable principal $B(G, A)$-bundle.

8.9. Remark. We used the assumption that $G$ is discrete and $(X, A)$ is triangulable at only one point-in showing (8.7) that $\left(B_{n}(G, X), B_{n}(G, X) \cap E_{n-1}\right)$ is an $N D R$ pair. The rest of the proof works for $G$ an arbitrary abelian topological group and $(X, A)$ an arbitrary closed based pair. It would be nice to get the conclusion of 
8.7-or at least of the theorem-under weaker hypotheses. One ought to be able to get a quasifibration theorem too, for the monoid case.

9. Classifying spaces. To begin, suppose $G$ is a monoid (not necessarily abelian). Then by 5.2(b) the inclusion $S^{0} \rightarrow I$ and the quotient map $I \rightarrow S^{1}, t \mapsto \bar{t}$, induce morphisms of monoids

$$
B\left(G, S^{0}\right) \longrightarrow B(G, I) \stackrel{p}{\longrightarrow} B\left(G, S^{1}\right) .
$$

Since $G$ is isomorphic to $B\left(G, S^{0}\right)$ under the correspondence $g \mapsto g l$, it is convenient to replace $B\left(G, S^{0}\right)$ by $G$, and the sequence $(9.1)$ becomes

$$
G \stackrel{i}{\longrightarrow} B(G, I) \stackrel{p}{\longrightarrow} B\left(G, S^{1}\right)
$$

where $i(g)=g 1$.

Now suppose $G$ is a topological monoid. For each $n \geqq 0$ define a diagram

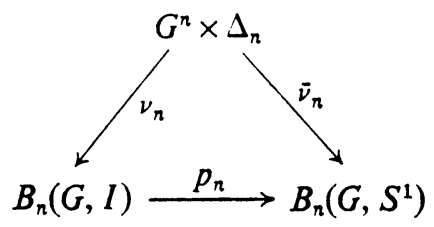

as follows: $\Delta_{n}$ is the subspace of the unit cube $I^{n}$ consisting of all $\left(t_{1}, \ldots, t_{n}\right)$ with $t_{1} \leqq \cdots \leqq t_{n} ; v_{n}\left(g_{1}, \ldots, g_{n}, t_{1}, \ldots, t_{n}\right)=g_{1} t_{1} \oplus \cdots \oplus g_{n} t_{n} ; p_{n}$ is the restriction of $p$; $\bar{\nu}_{n}=p_{n} \circ \nu_{n}$. Give $B_{n}(G, I)$ and $B_{n}\left(G, S^{1}\right)$ the quotient topologies from $v_{n}$ and $\bar{\nu}_{n}$, respectively. Note that then $p_{n}$ is also proclusive.

9.4. LEMMA. For each $n>0$, and for $X=I$ or $S^{1}, B_{n-1}(G, X)$ is a closed subspace of $B_{n}(G, X)$.

The proof can be based on 2.8, and is analogous to 6.2, but simpler. Because of the structure of $\Delta_{n}$, one can replace all the sets $Y_{j}, Y_{k l}$ of the proof of 6.2 by a singly indexed sequence of subsets of $G^{n} \times \Delta_{n}$.

9.5. Definition. If $G$ is a topological monoid and $X=I$ or $S^{1}$, then $B(G, X)$ shall have the topology of the union of the expanding sequence of spaces $B_{n}(G, X)$, where $B_{n}(G, X)$ has the quotient topology from (9.3).

9.6. Proposition. For $G$ and $X$ as in $9.5, B(G, X)$ is compactly generated.

Proof. This is analogous to 6.5. We prove by induction on $n$ that $B_{n}(G, X)$ is compactly generated. For the inductive step we use the fact (easily checked) that $v_{n}$ defines a relative homeomorphism

$$
\left(G^{n} \times \Delta_{n}, \nu_{n}^{-1} B_{n-1}(G, I)\right) \rightarrow\left(B_{n}(G, I), B_{n-1}(G, I)\right),
$$

with a similar statement for $\bar{\nu}_{n}$ and $S^{1}$. 
9.7. Remark. $B(G, I)$ and $B\left(G, S^{1}\right)$ have a monoid structure, but we do not claim it is continuous; see 9.19 . However, if $G$ is abelian, then by 9.18 and 6.6 this is true.

The following three propositions are analogous to (special cases of) 6.7 and 6.10 , and are easily proved.

9.8. Proposition (see (9.2)). $i: G \rightarrow B(G, I)$ is a closed imbedding and

is a proclusion.

$$
p: B(G, I) \rightarrow B\left(G, S^{1}\right)
$$

9.9. Proposition. If $\varphi: G \rightarrow G^{\prime}$ is a morphism of topological monoids and $X=I$ or $S^{1}$, then $\varphi_{*}: B(G, X) \rightarrow B\left(G^{\prime}, X\right)$ (defined in $\S 5$ ) is continuous.

9.10. Proposition. $B(G, I)$ is contractible, with contracting homotopy

$$
h_{s}: B(G, I) \rightarrow B(G, I)
$$

given by

$$
h_{s}\left(g_{1} t_{1} \oplus \cdots \oplus g_{n} t_{n}\right)=g_{1}\left(s t_{1}\right) \oplus \cdots \oplus g_{n}\left(s t_{n}\right) .
$$

Now we wish to imitate $\S 8$. The pair $(X, A)$ is now $\left(I, S^{0}\right)$ but the monoid $G$ may not be abelian. Nevertheless, a good deal of the set-theoretic and algebraic steps of $\S 8$ are still valid. Consequently we find it safe to omit proofs where the topological modifications are obvious.

Under the replacement $G \approx B\left(G, S^{0}\right)$, the action (8.2) becomes

$$
B(G, I) \times G \stackrel{\varphi}{\longrightarrow} B(G, I), \quad \varphi(u, g)=u \oplus g 1 .
$$

We cannot now rely on the continuity of the operation $\oplus$ in $B(G, I)$ to know that $\varphi$ is continuous, but this can be proved separately as follows: It suffices to show that the restriction of $\varphi$ to $B_{n}(G, I) \times G \rightarrow B_{n+1}(G, I)$ is continuous for each $n \geqq 0$. But this map can be put into the following diagram

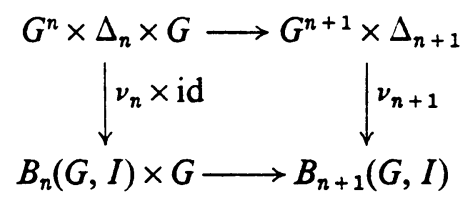

where the upper map sends $\left(g_{1}, \ldots, g_{n}, t_{1}, \ldots, t_{n}, g\right)$ to $\left(g_{1}, \ldots, g_{n}, g, t_{1}, \ldots, t_{n}, 1\right)$.

9.12. PROPOSITION (cf. 8.3). $p$ induces a homeomorphism from the orbit space $B(G, I) / G$ onto $B\left(G, S^{1}\right)$.

Corresponding to (8.4), define closed $G$-subspaces $E_{n}$ of $B(G, I)$ to be

$$
E_{n}=p^{-1} B_{n}\left(G, S^{1}\right)=B_{n}(G, I) \oplus i G .
$$

Note that now it happens that $E_{n-1}$ is contained in $B_{n}(G, I)$. 
9.13. Lemma (cf. 8.5). An element $g_{1} t_{1} \oplus \cdots \oplus g_{n} t_{n}$ of $B_{n}(G, I)$ is in $E_{n-1}$ if and only if (a) some $g_{j}=e$, or (b) some $t_{j}=0$ or 1 , or (c) $t_{j}=t_{k}$ for some $j \neq k$.

9.14. LEMMA (cf. 8.6). For each $n \geqq 0$ the action map $\varphi$ defines a relative homeomorphism

$$
\left(B_{n}(G, I), E_{n-1}\right) \times G \rightarrow\left(E_{n}, E_{n-1}\right) .
$$

The proof that $\varphi$ maps $B_{n}(G, I) \times G$ proclusively to $E_{n}$ is simpler than the corresponding part of 8.6 . One can easily apply 2.8 to the maps

$$
G^{n} \times \Delta_{n} \times G \stackrel{\varphi \circ\left(\nu_{n} \times \mathrm{id}\right)}{\longrightarrow} E_{n} \subset B_{n+1}(G, I) \stackrel{\nu_{n+1}}{\longleftarrow} G^{n+1} \times \Delta_{n+1} .
$$

9.15. LEMMA. If $G$ is a nicely based topological monoid, then for each $n \geqq 0$, $\left(B_{n}(G, I), E_{n-1}\right)$ is an NDR pair.

Proof. For $n=0$ we have $\left(B_{0}(G, I), E_{-1}\right)=(\{e\}, \varnothing)$. Suppose, inductively, that $n \geqq 0$ and $\left(B_{n}(G, I), E_{n-1}\right)$ is an $N D R$ pair. Consider the diagram

$$
\begin{aligned}
& G^{n} \times \Delta_{n} \times G \times I \stackrel{\tilde{\psi}}{\longrightarrow} G^{n+1} \times \Delta_{n+1} \\
& \underset{B_{n}(G, I) \times G \times I \stackrel{\psi}{\nu_{n} \times \mathrm{id} \times \mathrm{id}}{ }^{\longrightarrow} B_{n+1}(G, I),}{\downarrow}
\end{aligned}
$$

where $\psi\left(g_{1}, \ldots, g_{n}, t_{1}, \ldots, t_{n}, g, t\right)=\left(g_{1}, \ldots, g_{n}, g, t_{1} t, \ldots, t_{n} t, t\right)$, and where $\psi$ is well defined by requiring commutativity. $\psi$ is a proclusion because the other three maps in the diagram are proclusions. Using 9.13 , it is easy to see that $\psi$ defines a relative homeomorphism

$$
\left(B_{n}(G, I), E_{n-1}\right) \times(G, e) \times\left(I, S^{0}\right) \rightarrow\left(B_{n+1}(G, I), E_{n}\right) .
$$

Since all three factors of the product are $N D R$ pairs, the result then follows from Theorem 6.3 and Lemma 8.4 of [11].

If in addition to the hypothesis of $9.15, G$ is a topological group, then by 9.14 and 9.15 , we can apply Theorem 4.2 to $B(G, I)$. This, in combination with 9.12 and 9.10 , gives:

9.17. THEOREM (cf. [7], [12]). If $G$ is a nicely based topological group, then

$$
\left(B(G, I), p, B\left(G, S^{1}\right)\right)
$$

is a numerable principal $G$-bundle, and $B(G, I)$ is contractible. Hence $B\left(G, S^{1}\right)$ is a classifying space for $G$.

9.18. THEOREM. If $G$ is an abelian topological monoid and $X=I$ or $S^{1}$, then the topology on $B(G, X)$ defined in 9.5 agrees with the topology defined in 6.3.

Proof. By $6.7\left(\right.$ b) and 9.8 , the same function $B(G, I) \rightarrow B\left(G, S^{1}\right)$ is a proclusion for both topologizations. Hence it suffices to prove the result for $B(G, I)$. For this 
it suffices to show that for each $n \geqq 0, B_{n}(G, I)$ gets the same quotient topology by both methods. Let $B_{n}^{u}(G, I)$ and $B_{n}^{v}(G, I)$ denote $B_{n}(G, I)$ with the topologies from $\mu_{n}$ and $\nu_{n}$ respectively, and let $i: B_{n}^{v}(G, I) \rightarrow B_{n}^{\mu}(G, I)$ be the identity function. It is convenient to think of $\mu_{n}$ as being defined on $G^{n} \times I^{n}$, through the obvious homeomorphism $(G \times I)^{n} \approx G^{n} \times I^{n}$. Then clearly $i$ is continuous, since $G^{n} \times \Delta_{n} \subset G^{n} \times I^{n}$.

To see that $i$ is closed, we apply 2.8 to the maps $\left(v_{n}, i, \mu_{n}\right)$. For each $\alpha \in S_{n}$ (see (6.4)), let $I_{\alpha}^{n}=\left\{t \in I^{n}: t \cdot \alpha \in \Delta_{n}\right\}$. Then, since $G$ is abelian, we get commutative diagrams

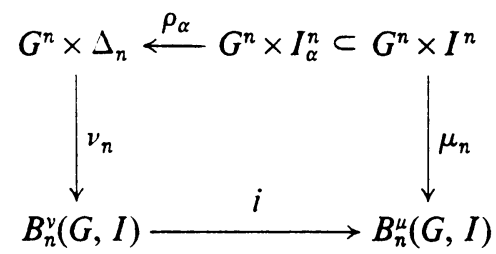

where $\rho_{\alpha}(g, t)=(g \cdot \alpha, t \cdot \alpha)$. It is easy to check the hypotheses of 2.8 , so that $i$ is closed and the proof is complete.

9.19. RemARKS. In treating $B(G, X)$ in $\S 6$ we assumed $G$ to be abelian because some of the proofs depend on that assumption. But if $G$ is an arbitrary topological monoid and $X$ is a based space, the functions $\mu_{n}$ of (6.1) are still defined and could be used to topologize $B(G, X)$. So it makes sense to ask whether it is really necessary to assume that $G$ is abelian in Theorem 9.18. The following example shows that it is. Let $G$ be a discrete, nonabelian group. Suppose that the topology on $B\left(G, S^{1}\right)$ defined from the $\mu_{n}$ agrees with that defined in the present section. Then the proof of 6.7 still applies to show that $B\left(G, S^{1}\right)$ is a topological monoid. Hence $\pi_{1} B\left(G, S^{1}\right)$ is abelian. But by $9.17, \pi_{1} B\left(G, S^{1}\right) \approx \pi_{0} G \approx G$, which is nonabelian. Incidentally, the first paragraph of the proof of 9.18 then shows that the topology on $B\left(G, S^{1}\right)$ of the present section is strictly larger than that defined by the $\mu_{n}$.

10. The canonical $H$-isomorphisms $B(G, X) \rightarrow \Omega B(G, S X)$. If $G$ is a topological monoid, we give $B\left(G, S^{1}\right)$ the topology of $\S 9$, and we define a map

$$
G \stackrel{\eta}{\longrightarrow} \Omega B\left(G, S^{1}\right), \quad \eta(g)(t)=g(\overline{1-t}) .
$$

10.2. THEOREM. If $G$ is a nicely based topological group then the map (10.1) is an $H$-isomorphism. (It is a morphism of $H$-spaces and also is a based homotopy equivalence.)

Proof. Let $E_{p}$ be the mapping track (§3) of the projection $p: B(G, I) \rightarrow B\left(G, S^{1}\right)$ and consider the diagram

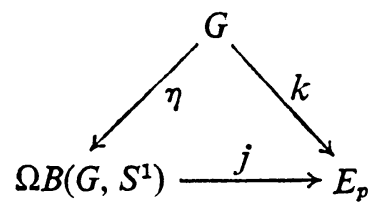


where $k(g)=(g 1, *)$ and where $j$ is as in 3.1. By $9.8, k$ is essentially the standard inclusion of the fiber of $p$ into $E_{p}$. Since $p$ is a based fibration by $9.17, k$ is a based homotopy equivalence. Passing to based homotopy classes, we remark that it is a standard fact that $[k]^{-1}[j]$ is a morphism of $H$-spaces. (This works for any numerable principal $G$-bundle.) Since $B(G, I)$ is contractible, $[j]$ is invertible by 3.1 , so that $[k]^{-1}[j]$ is an $H$-isomorphism. If we can show that $[j][\eta]=[k]$, we will be finished, for then $[\eta]=[j]^{-1}[k]$ is an $H$-isomorphism. But to see that $k \simeq j \circ \eta$ we define a homotopy $k_{s}: G \rightarrow E_{p}$ by $k_{s}(g)=\left(g(1-s),(\eta(g))^{s}\right)$, where

$$
(\eta(g))^{s}(t)=\eta(g)(s t)=g(\overline{1-s t}) .
$$

If $G$ is an abelian topological monoid and $X$ is a based space, then we give $B(G, X)$ the topology of $\S 6$, and we define a map

$$
\begin{aligned}
B(G, X) & \stackrel{h}{\longrightarrow} \Omega B(G, S X), \\
h\left(g_{1} x_{1} \oplus \cdots \oplus g_{n} x_{n}\right)(t) & =g_{1}\left(\bar{t} \wedge x_{1}\right) \oplus \cdots \oplus g_{n}\left(\bar{t} \wedge x_{n}\right) .
\end{aligned}
$$

10.4. THEOREM. If $G$ is an abelian topological group, $X$ is a based space, and $B(G, X)$ is nicely based, then the map (10.3) is an H-isomorphism.

Proof. Let $\tau: S X \rightarrow X \wedge S^{1}$ be the homeomorphism given by

$$
\tau(\bar{t} \wedge x)=x \wedge \overline{1-t}
$$

Consider the diagram

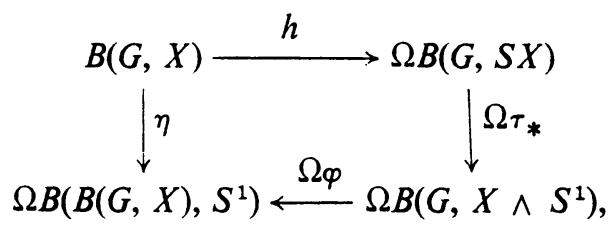

where $\eta$ is the map of $(10.1)$ and $\varphi$ is the homeomorphism of 6.13. The diagram is easily seen to commute. By 10.2 and $9.18, \eta$ is an $H$-isomorphism. But $\Omega \varphi$ and $\Omega \tau_{*}$ are $H$-isomorphisms since $\varphi$ and $\tau_{*}$ are homeomorphisms. Thus $h$ is the composite of three $H$-isomorphisms.

10.5. COROLlaRY. If $G$ is a discrete abelian group and $X$ is a based space having the homotopy type of a CW complex, then the map (10.3) is an H-isomorphism.

Proof. By [9] there exists a based homotopy equivalence $f: X \rightarrow X^{\prime}$, where $X^{\prime}$ is triangulable. We get a commutative diagram

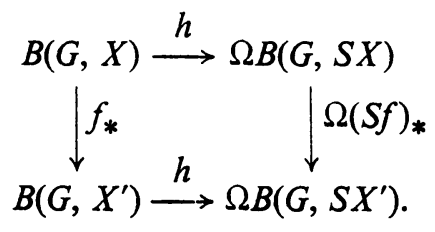


By $\S 7, B\left(G, X^{\prime}\right)$ is nicely based, so by 10.4 the lower $h$ is an $H$-isomorphism. By 6.7 and 6.10 the two vertical maps are also $H$-isomorphisms; hence the result.

We remark that for this corollary one can also use 8.8 as a basis of proof. The next corollary is obtained inductively, beginning with the isomorphism

$$
B\left(G, S^{0}\right) \approx G \text {. }
$$

10.6. CoROllaRY. If $G$ is a discrete abelian group and $n \geqq 0$, then $B\left(G, S^{n}\right)$ is an Eilenberg-MacLane space $K(G, n)$.

11. The homology-cohomology modules $[X, B(G, Y)]$. Throughout this section let $R$ be a fixed commutative ring with unit. All modules and their tensor products are taken over $R$. A topological module $M$ is an abelian topological group which is also a module such that the multiplication $R \times M \rightarrow M$ is continuous. If $X$ is a based space, then $[X, M]$ has an obvious module structure.

If $G$ is a topological module and $Y$ is a based space, then $B(G, Y)$ is a topological module: if $r \in R$ and $u \in B(G, Y)$ then $r \cdot u$ is defined by $(r \cdot u)(y)=r \cdot u(y)$. Note then that $r \cdot\left(g_{1} y_{1} \oplus \cdots \oplus g_{n} y_{n}\right)=\left(r g_{1}\right) y_{1} \oplus \cdots \oplus\left(r g_{n}\right) y_{n}$. From this and 6.3 it is obvious that $R$-multiplication is continuous.

We are interested in the modules $[X, B(G, Y)]$ where $X$ and $Y$ are based spaces and $G$ is a discrete module. It follows from 6.9 that $[\cdot, B(\cdot, \cdot)]$ is a trifunctor, contravariant in the first variable and covariant in the second and third. Trivially, homotopic based maps in the first variable induce equal homomorphisms. By 6.10 a similar statement holds for the third variable.

We define the suspension morphism $\sigma$ to be the composite

$$
\sigma:[X, B(G, Y)] \stackrel{h_{*}}{\longrightarrow}[X, \Omega B(G, S Y)] \approx[S X, B(G, S Y)],
$$

where $h$ is the canonical map (10.3).

11.2. Proposition. $\sigma$ is a morphism of modules. If $Y$ has the homotopy type of a $C W$ complex, then $\sigma$ is an isomorphism.

Proof. The first statement is easily seen from the following property of $h$ : if $r_{1}, r_{2} \in R, u_{1}, u_{2} \in B(G, Y)$, and $t \in I$, then the equation

$$
\left(h\left(r_{1} \cdot u_{1} \oplus r_{2} \cdot u_{2}\right)\right)(t)=r_{1} \cdot\left(h\left(u_{1}\right)(t)\right) \oplus r_{2} \cdot\left(h\left(u_{2}\right)(t)\right)
$$

holds in the module $B(G, S Y)$. The second statement follows from 10.5 .

The following proposition describes an exactness property of the functor $[X, B(G, \cdot)]$, where $X$ is a fixed based space and $G$ is a fixed (discrete) module.

11.3. Proposition. If $\left(Y, Y^{\prime}\right)$ is a based pair having the homotopy type of a $C W$ pair, then the sequence

$$
\left[X, B\left(G, Y^{\prime}\right)\right] \rightarrow[X, B(G, Y)] \rightarrow\left[X, B\left(G, Y / Y^{\prime}\right)\right]
$$

is exact. 
Proof. By [9] and 6.10 we may assume that $\left(Y, Y^{\prime}\right)$ is triangulable. Then, by $8.8, B\left(G, Y^{\prime}\right) \rightarrow B(G, Y) \rightarrow B\left(G, Y / Y^{\prime}\right)$ is a based fibration, whence the result.

One could also state a dual, somewhat more generally valid, exactness property for the functor $[\cdot, B(G, Y)]$.

For sufficiently nice $X$ and $Y$ one can describe the modules $[X, B(G, Y)]$ in terms of the (singular) cohomology of $X$ and the homology of $Y$. First, using 11.2 and 11.3, and proceeding much as in $\S 6$ of Dold and Thom [4], one gets the following theorem.

11.4. THEOREM. If $Y$ is a based space having the homotopy type of a CW complex, then $\left[S^{n}, B(G, Y)\right]$ is isomorphic to the reduced homology module $\tilde{H}_{n}(Y ; G)$.

We comment that the coefficient module is $G$ since $B\left(G, S^{0}\right) \approx G$. Next, using 11.4 as a description of the homotopy groups of $B(G, Y)$ and applying Moore's theorem [2] or [4] that a topological abelian group has the homotopy type of a product of Eilenberg-MacLane spaces, one gets the following theorem. It can be viewed as a generalization of a theorem of Burghelea and Deleanu [1].

11.5. THEOREM. If $X$ and $Y$ are based spaces having the homotopy type of a $C W$ complex, then

$$
[X, B(G, Y)] \approx \prod_{n=0}^{\infty} \tilde{H}^{n}\left(X ; \tilde{H}_{n}(Y ; G)\right)
$$

Now we want to show how the construction $B(\cdot, \cdot)$ can be used to define and treat directly cross product pairings in the homology-cohomology modules $[X, B(G, Y)]$.

11.6. Proposition AND Definition. If $G$ and $G^{\prime}$ are modules and $Y$ and $Y^{\prime}$ are based spaces, then there is a continuous bilinear map

$$
B(G, Y) \times B\left(G^{\prime}, Y^{\prime}\right) \rightarrow B\left(G \otimes G^{\prime}, Y \wedge Y^{\prime}\right)
$$

given by

$$
\left(\sum_{i} g_{i} y_{i}, \sum_{j} g_{j}^{\prime} y_{j}^{\prime}\right) \mapsto \sum_{i, j}\left(g_{i} \otimes g_{j}^{\prime}\right)\left(y_{i} \wedge y_{j}^{\prime}\right)
$$

Proof. It is easy to check that the map is well defined and bilinear. Continuity can be seen by making a commutative diagram

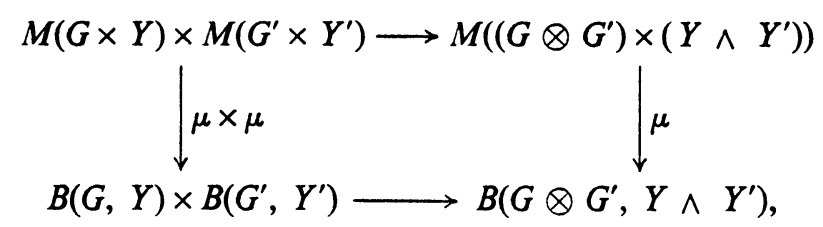

where $\mu$ is the proclusion of (6.12), and by applying 2.2 .

Since the map of 11.6 is bilinear, it can also be viewed as a map

$$
B(G, Y) \wedge B\left(G^{\prime}, Y^{\prime}\right) \rightarrow B\left(G \otimes G^{\prime}, Y \wedge Y^{\prime}\right)
$$


Given also based spaces $X$ and $X^{\prime}$, we can now define our generalized cross product pairing

$$
[X, B(G, Y)] \times\left[X^{\prime}, B\left(G^{\prime}, Y^{\prime}\right)\right] \stackrel{\wedge}{\longrightarrow}\left[X \wedge X^{\prime}, B\left(G \otimes G^{\prime}, Y \wedge Y^{\prime}\right)\right]
$$

as the composite of the two functions

$$
\begin{aligned}
{[X, B(G, Y)] \times\left[X^{\prime}, B\left(G^{\prime}, Y^{\prime}\right)\right] } & \rightarrow\left[X \wedge X^{\prime}, B(G, Y) \wedge B\left(G^{\prime}, Y^{\prime}\right)\right] \\
& \rightarrow\left[X \wedge X^{\prime}, B\left(G \otimes G^{\prime}, Y \wedge Y^{\prime}\right)\right],
\end{aligned}
$$

where the first function sends $\left([f],\left[f^{\prime}\right]\right)$ to $\left[f \wedge f^{\prime}\right]$, and the second is induced by (11.7).

Bilinearity of (11.8) is straightforward to check. Also associativity, in the obvious sense, can be seen easily from the (strict!) associativity that (11.7) enjoys.

The product (11.8) is well related to suspension (11.1) through the following commutative diagram. Let $\iota \in\left[S^{1}, B\left(R, S^{1}\right)\right]$ be the homotopy class of the map $s \mapsto 1 s$. (It is easy to see from 10.5 that $\left[S^{1}, B\left(R, S^{1}\right)\right] \approx R$, and $\iota$ is a generator.) The diagram is

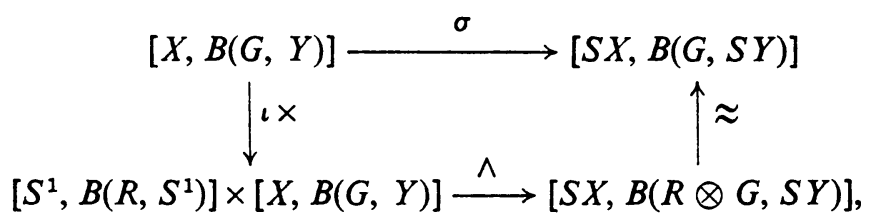

where the second vertical map is induced by the standard isomorphism $R \otimes G \rightarrow G$. Commutativity is straightforward to check from the definitions.

Before establishing commutativity properties of the product (11.8) we give two lemmas. The first is elementary and standard. The second one uses the structure of $B(G, X)$ in an explicit and interesting way.

11.10. Lemma. Let $p \geqq 2$ and let $\varphi: S^{p} \rightarrow S^{p}$ be a map that switches two factors of $S^{p}=S^{1} \wedge \cdots \wedge S^{1}$. Then $\varphi \simeq \psi$ where

$$
\psi\left(\bar{t}_{1} \wedge \bar{t}_{2} \wedge \cdots \wedge \bar{t}_{p}\right)=\overline{1-t_{1}} \wedge \bar{t}_{2} \wedge \cdots \wedge \bar{t}_{p}
$$

Hence for any based space $W, \varphi^{*}=-\mathrm{id}:\left[S^{p}, W\right] \rightarrow\left[S^{p}, W\right]$.

11.11. Lemma. If $\varphi: S^{p} \rightarrow S^{p}$ is a map switching two factors and $G$ is a module, then $\varphi_{*} \simeq$-id: $B\left(G, S^{p}\right) \rightarrow B\left(G, S^{p}\right)$.

Proof. Let $\psi$ be as in 11.10. Since $\varphi_{*} \simeq \psi_{*}$, it suffices to show $\psi_{*} \simeq-$ id. Define homotopies $\alpha_{s}, \beta_{s}: I \rightarrow I$ by $\alpha_{s}(t)=(1-t) s$ and $\beta_{s}(t)=(1-t) s+t$. Then define a homotopy $h_{s}: B\left(G, S^{p}\right) \rightarrow B\left(G, S^{p}\right)$ by

$$
h_{s}\left(\sum_{i} g_{i}\left(\bar{t}_{i} \wedge u_{i}\right)\right)=\sum_{i}\left(g_{i}\left(\alpha_{s}\left(t_{i}\right) \wedge u_{i}\right)-g_{i}\left(\bar{\beta}_{s}\left(t_{i}\right) \wedge u_{i}\right)\right),
$$


for $s, t_{i} \in I, g_{i} \in G, u_{i} \in S^{p-1}$. Since

$$
\overline{\alpha_{s}(t)}=\overline{\beta_{s}(t)} \text { for } t \in S^{0},
$$

$h_{s}$ is well defined and continuous. One checks that $h_{0}=-\mathrm{id}$ and $h_{1}=\psi_{*}$. This completes the proof.

Now suppose we are given based spaces $X, X^{\prime}, Y, Y^{\prime}$, and modules $G, G^{\prime}$. Let $\tau_{1}: X^{\prime} \wedge X \rightarrow X \wedge X^{\prime}, \tau_{2}: G \otimes G^{\prime} \rightarrow G^{\prime} \otimes G$, and $\tau_{3}: Y \wedge Y^{\prime} \rightarrow Y^{\prime} \wedge Y$ be the twisting morphisms. The general commutativity property for the product (11.8) is expressed by the commutativity of the following diagram, which is straightforward to verify from the definitions:

$$
\begin{aligned}
& {[X, B(G, Y)] \times\left[X^{\prime}, B\left(G^{\prime}, Y^{\prime}\right)\right] \stackrel{\wedge}{\longrightarrow}\left[X \wedge X^{\prime}, B\left(G \otimes G^{\prime}, Y \wedge Y^{\prime}\right)\right]} \\
& \downarrow \text { switch } \downarrow\left[\tau_{1}, B\left(\tau_{2}, \tau_{3}\right)\right] \\
& {\left[X^{\prime}, B\left(G^{\prime}, Y^{\prime}\right)\right] \times[X, B(G, Y)] \longrightarrow\left[X^{\prime} \wedge X, B\left(G^{\prime} \otimes G, Y^{\prime} \wedge Y\right)\right]}
\end{aligned}
$$

Now consider the special case $X=S^{m}, X^{\prime}=S^{n}$, so that we are dealing with homology. Then we identify $X \wedge X^{\prime}=S^{m+n}=X^{\prime} \wedge X$. We write $\tau_{1}: S^{m+n} \rightarrow S^{m+n}$ as the composition of $m n$ switches of $S^{1}$ factors, and apply 11.10 to each of these. The result is that in (11.12) we can replace $\left[\tau_{1}, B\left(\tau_{2}, \tau_{3}\right)\right]$ by $(-1)^{m n}\left[\mathrm{id}, B\left(\tau_{2}, \tau_{3}\right)\right]$. Then (11.12) expresses the standard skew commutativity of the homology cross product.

Similarly, conisider the special case $Y=S^{m}, Y^{\prime}=S^{n}$, so that we are dealing with cohomology. An analogous application of $11.11 \mathrm{mn}$ times allows us to replace $\left[\tau_{1}, B\left(\tau_{2}, \tau_{3}\right)\right]$ in $(11.12)$ by $(-1)^{m n}\left[\tau_{1}, B\left(\tau_{2}\right.\right.$, id $\left.)\right]$. Then (11.12) expresses the standard skew commutativity of the cohomology cross product.

\section{BIBLIOGRAPHY}

1. D. Burghelea and A. Deleanu, On certain two-space homology-cohomology groups, Rev. Roumaine Math. Pures Appl. 11 (1966), 703-712.

2. H. Cartan, Séminaire 7: Algèbres d'Eilenberg-MacLane et homotopie, Paris, 1955.

3. A. Dold, Partitions of unity in the theory of fibrations, Ann. of Math. 78 (1963), 223-255.

4. A. Dold and R. Thom, Quasifaserungen und unendliche symmetrische Produkte, Ann. of Math. 67 (1958), 239-281.

5. S. Eilenberg and N. Steenrod, Foundations of algebraic topology, Princeton Univ. Press, Princeton, N. J., 1952.

6. W. Hurewicz, On the concept of fiber space, Proc. Nat. Acad. Sci. U.S.A. 41 (1955), 956-961.

7. R. Milgram, The bar construction and abelian H-spaces, Illinois J. Math. 11 (1967), 242-250.

8. J. Milnor, Construction of universal bundles. I, Ann. of Math. 63 (1956), 272-284.

9. - On spaces having the homotopy type of a CW-complex, Trans. Amer. Math. Soc. 90 (1959), 272-280.

10. M. Rothenberg and N. E. Steenrod, The cohomology of classifying spaces of H-spaces, Bull. Amer. Math. Soc. 71 (1965), 872-875. 
11. N. E. Steenrod, A convenient category of topological spaces, Michigan Math. J. 14 (1967), 133-152.

12. - Milgram's classifying space of a topological group, Topology 7 (1968), 349-368.

INSTITUTE FOR ADVANCED STUDY,

Princeton, NeW Jersey

UNIVERSITY OF GeORGIA,

Athens, Georgia 